\title{
Control Optimisation of a Pump-Controlled Hydraulic System using Digital Displacement Pumps
}

\author{
L. Viktor Larsson, Robert Lejonberg*, and Liselott Ericson \\ Division of Fluid and Mechatronic Systems (Flumes), Department of Management and Engieering (IEI), \\ Linköping University, Linköping, Sweden \\ E-mail: viktor.larsson@liu.se; robert.lejonberg@epiroc.com; liselott.ericson@liu.se \\ *Epiroc Rock Drills AB, Örebro, Sweden
}

\begin{abstract}
When dealing with electrification of working machines, energy-efficient operation is key to maximise the usage of the limited capacity of on-board batteries. Previous research indicate that plenty is gained by reducing component and system losses by means of design. In contrast, this paper focuses on how to maximise energy efficiency by means of control optimisation. Dynamic programming with backward-facing simulation is used to find the optimal electric motor speed trajectory for a scooptram machine equipped with pump control, using digital displacement pumps with dynamic flow sharing as enabling technology. The results show that low shaft speed is preferred to minimise drag losses from parasitic components, partly facilitated by the relatively high and operation point-independent efficiencies of the pumps and electric motor. The results indicate energy reduction of $5-9 \%$, where higher figures could be expected for other, more hydraulic-intense applications, such as excavators.
\end{abstract}

Keywords: Control Optimisation, Pump Control, Digital Displacement Pump, Mobile Hydraulics

\section{Introduction}

Mining machines are mobile working machines used to move ore and other granular material in mines. Currently, these machines are subject to electrification, with decreased global use of energy and fossil fuels as primary motivators. For mining machines, a lowered energy consumption is directly related to cost for the user, but electrification also has positive side effects. By replacing the conventionally used combustion engine with an electric power source, local emissions such as exhaust gases, heat and noise are reduced. This reduction, or even elimination, improves the working environment for the machine operator and reduces the need for ventilation, which is a major energy consumer in a mine.

For electrified system solutions with on-board batteries, limited energy capacity compared to diesel fuel puts high requirements on the energy efficiency of the machine's motion system. This aspect is challenging in particular for the work functions, that conventionally are powered by a hydraulic system with throttle control. In pump-controlled hydraulic systems, valve throttling is minimised by powering each function with an individual pump [1].

Traditionally, pump-controlled systems have been difficult to motivate as they require the pumps to be dimensioned for the maximum flow of each function. With several functions, this results in an expensive system with multiple large pumps that primarily operate at part load with poor efficiency [2]. In this paper, the use of a digital displacement pump (DDP) is considered as a measure to mitigate the above-mentioned drawbacks of pump control.

\subsection{Scope and Delimitations}

The aim of the paper is to, in terms of energy efficiency, evaluate the potential of the concept presented in section 2 applied to the Epiroc ST14 Battery, and to investigate the gains of optimally controlling the electric motor shaft speed. The work functions considered are boom, bucket and steering while the component sizing and the driveline are not within the scope of the paper. The current available DDP version from Danfoss is considered, which operates in pump-mode only [3]. Energy recuperation from the loads is thus not considered. 


\subsection{Scooptram}

The mining machine considered in this paper is referred to as scooptram. Similar to a wheel loader, a scooptram uses articulated steering and has a loader with boom and bucket. In contrast to the wheel loader a scooptram is, however, designed to be used under ground, and therefore has stricter space requirements, which results in a rather compact design. The specific machine model studied in this paper is the Epiroc ST14 Battery [4], shown in figure 1. It has a battery (Li-Ion NMC) as primary energy source, which is swapped to a recharged battery when empty. This concept allows battery charging from the grid and machine operation to occur simultaneously. The ST14 Battery uses one electric motor (traction motor) for propulsion and another (auxiliary motor) to power the work functions (boom, bucket and steering). Today, the work functions are implemented with a conventional load sensing system with two axial-piston pumps connected in parallel. This paper explores the potential of replacing each axial-piston pump with a Danfoss Digital Displacement Pump (DDP) [3], shown in figure 2.

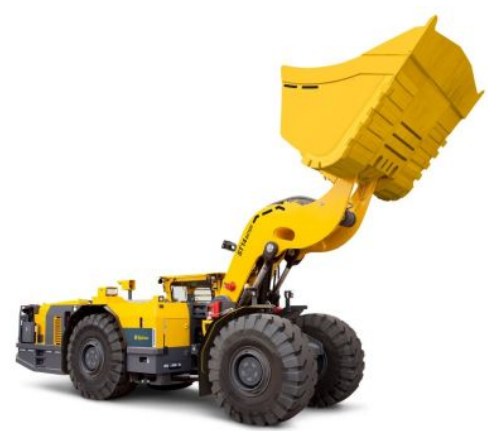

(a) Epiroc ST14 Battery.

\begin{tabular}{lcc}
\hline Property & Value & Unit \\
\hline Mass & 42 & Tonnes \\
Nominal power (traction motor) & 200 & $\mathrm{~kW}$ \\
Nominal power (auxiliary motor) & 160 & $\mathrm{~kW}$ \\
\hline
\end{tabular}

(b) Machine Data [4].

Figure 1: Scooptram application considered in the paper.

\subsection{Digital Displacement Pump}

A DDP is a piston pump where the flow is controlled by individually connecting and disconnecting each piston to the pump's high and low pressure sides, enabled by actively controlled high-speed solenoid valves [5]. The pump displacement is then varied by controlling the number of active and non-active pump cylinders during one or several shaft revolutions [3]. Within the scope of this paper, it is assumed that this property is equivalent to a continuously variable displacement in a conventional pump.

A benefit with the DDP design is that it results in significantly higher part load efficiencies compared to conventional axial piston pumps. This benefit was showcased in the 16-tonnes DEXTER excavator, where the conventional pumps were replaced with DDPs. This swap resulted in fuel savings of up to $20 \%$ with maintained productivity [6].

Another attractive feature of the DDP is that its physical layout facilitates access to the individual pistons. One pump can thus be treated as several pumps connected in parallel on the same shaft. Each pump, referred to as pumplet, can in turn be dedicated to an individual function [7]. If each pumplet flow is individually controlled, a system solution classified as a centralised pump-controlled system with mechanical power distribution [1] can thereby be achieved. In this paper, in contrast to [7], the DDPs are powered by an electric motor with variable speed, which presents a degree of freedom.

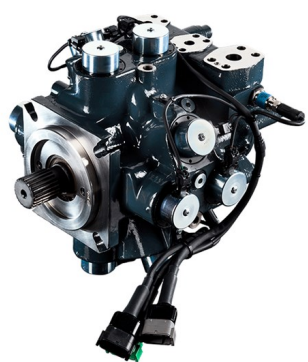

(a) Danfoss Digital Displacement Pump (DDP).

\begin{tabular}{lccc}
\hline Property & & Value & Unit \\
\hline Max displacement & $D_{P}$ & 96 & $\mathrm{~cm}^{3} /$ revolution \\
Number of pumplets & & 4 & \\
Pumplet displacement & $D_{p}$ & 24 & $\mathrm{~cm}^{3} /$ revolution \\
Pistons per pumplet & & 3 & \\
Piston volume & & 8 & $\mathrm{~cm}^{3}$ \\
\hline
\end{tabular}

(b) Size data (one unit) [3].

Figure 2: Danfoss Digital Displacement Pump (DDP). 
From a control perspective, a pump with variable speed and variable displacement has a degree of freedom since the pump flow is the product of these two variables. Traditionally, this freedom has been locked by considering either constant speed or constant displacement, primarily due to cost reasons [1]. One recent exception is [8], where a speed-controlled pump with discretely variable displacement is considered. As previously mentioned, this paper assumes continuously variable pumplet displacements.

\subsection{Dynamic Programming}

To lock the degree of freedom, optimal control is considered in this paper, using deterministic Dynamic Programming (DP). With DP, the control problem is discretised in state-time, where a recorded drive cycle is used to find the optimal control decision for each discrete time instant of the cycle, proceeding backward in time. The primary benefit with DP is that it yields the globally optimal solution (for a given discretisation). This benefit does, however, come with two important drawbacks. The first is a high computational cost, that increases exponentially with the number of states and control signals considered. The other is that it requires knowledge of the complete cycle, which means that the obtained control strategy is not implementable in practice. Rather, the results from the DP optimisation can be used to develop and evaluate causal control strategies. This is common practice within research of hybrid vehicles, where a DP result is commonly used as benchmark when evaluating energy management strategies, see for instance [9]. In this paper, the use of DP serves primarily two purposes:

- To explore the maximum potential of the considered system concept.

- To provide knowledge on how to optimally control the concept in a future implementation.

\section{System Concept}

Figure 3 shows the concept investigated in the paper. The conventional pumps in the ST14 Battery are replaced with DDPs $\left(P_{1}\right.$ and $\left.P_{2}\right)$ connected to two valve blocks, while the rest of the system is unchanged. The three loads are controlled with directional valves $\left(V_{1}-V_{3}\right)$ that are assumed fully open when the load cylinders are in motoring mode and are used for meter-out throttling when the load cylinders are in pumping mode. The boom and bucket functions have bypass valves that are assumed to be controlled simultaneously with the directional valve of each function, to redirect some of the flow from the piston side chamber to the piston rod side chamber when the cylinder is in pumping mode, thereby reducing the required pump flow.

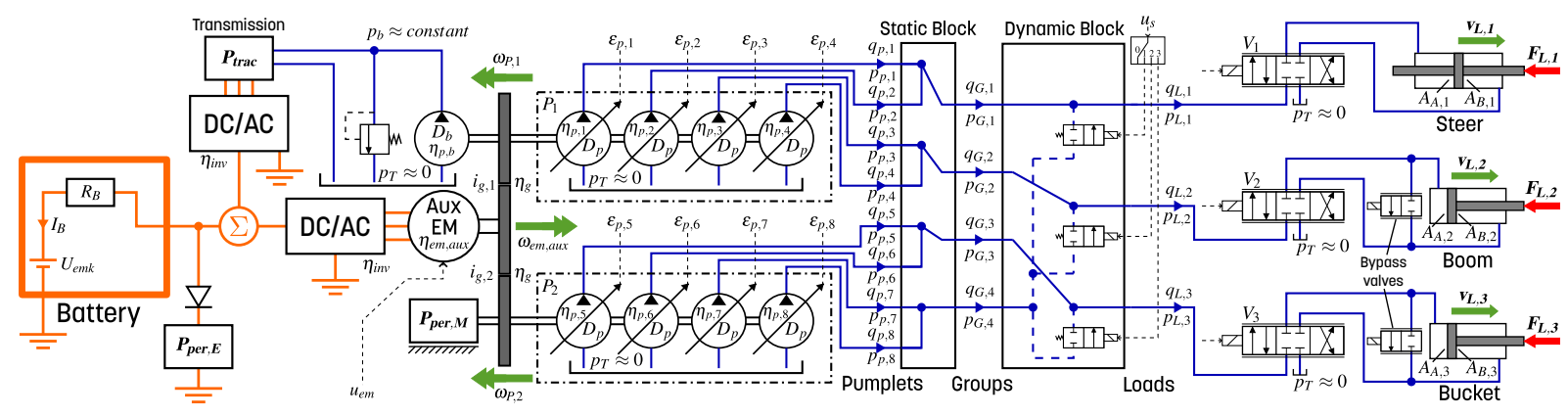

Figure 3: Layout of the system to be optimised. States that are determined directly by the drive cycle in the optimisation are highlighted in bold. Any check valves for load holding purposes are omitted in the figure.

The pumplets are connected to a static block which combines the pumplets into groups. The dynamic block connects the pumplet groups to the loads. In contrast to the static block, the dynamic block can change during use of the system, thereby enabling dynamic flow or pumplet allocation [7]. The idea of the concept is to dedicate group 1, 2 and 3 to load 1, 2 and 3, respectively, and have group 4 available as a shared resource. For instance, if $100 \mathrm{l} / \mathrm{min}$ is required by load 1 , but group 1 can only provide $50 \mathrm{l} / \mathrm{min}$, group 4 can provide the remaining 50 $1 / \mathrm{min}$. This enables moderate pumplet sizes as the loads seldom require their maximum flow simultaneously for the considered application.

The DDPs are powered by the auxiliary electric motor (Aux EM) which also powers peripheral mechanical functions $\left(P_{p e r, M}\right.$, e.g. brakes, cooling) and the transmission clutch boost circuit, which consists of a fixed displacement gear pump $\left(D_{b}\right)$ connected to a pressure relief valve. The auxiliary and traction $\left(P_{\text {trac }}\right)$ electric motors are powered by the battery which also powers peripheral electric functions $\left(P_{p e r, E}\right.$, e.g. A/C, battery cooling).

As previously discussed, the speed of the auxiliary electric motor is a free variable, and there is a compromise to make between shaft speed and flow sharing. This compromise is found using control optimisation in this paper. 


\section{Problem Formulation}

The control optimisation problem for the considered concept is formulated as:

$$
\begin{aligned}
& \underset{u_{e m}(t)}{\operatorname{minimize}} E_{B}\left(u_{e m}(t), \bar{\Lambda}(t)\right) \\
& \text { subject to } \\
& 0 \leq \varepsilon_{p, k}(t) \leq 1, \quad k=1,2, \ldots, z_{p}, \\
& u_{s}(t)=j, \quad j \in\left[0,1, \ldots, z_{L}\right], \\
& u_{\text {em,min }} \leq u_{\text {em }}(t) \leq u_{\text {em, } \max }, \\
& I_{B, \min }(t) \leq I_{B}(t) \leq I_{B, \max }(t), \\
& \omega_{\text {em,aux,min }}(t) \leq \omega_{\text {em,aux }}(t) \leq \omega_{\text {em,aux,max }}, \\
& \dot{\omega}_{\text {em,aux }}(t)=u_{\text {em }}(t) \\
& \omega_{\text {em,aux }}\left(t_{0}\right)=\omega_{\text {em,aux }, \min }\left(t_{0}\right), \\
& \omega_{\text {em,aux }, \min }\left(t_{f}\right) \leq \omega_{\text {em,aux }}\left(t_{f}\right) \leq \omega_{\text {em,aux,max }}
\end{aligned}
$$

where $u_{e m}(t)$ is the control signal, which is the angular acceleration of the auxiliary electric motor. This choice of control signal is made to enable limitation in shaft acceleration and thus avoid solutions with undesired and unrealistic shattering of the motor speed. It may also be noted that the introduction of the angular acceleration defines the angular speed as a state, which makes the optimal solution time-dependant. From a strict mathematical perspective, $u_{e m}(t)$ may also be interpreted as an additional degree of freedom. $\bar{\Lambda}(t)$ is the drive cycle:

$$
\bar{\Lambda}(t)=\left[\begin{array}{c}
F_{L, i}(t) \\
v_{L, i}(t) \\
\vdots \\
F_{L, z_{L}}(t) \\
v_{L, z_{L}}(t) \\
P_{\text {trac }}(t) \\
\omega_{\text {em,aux }, \text { min }}(t)
\end{array}\right] \text {, with } t \in\left[t_{0}, t_{f}\right]
$$

where $F_{L, i}(t)$ and $v_{L, i}(t)$ is the force and velocity of load $i$, respectively. $P_{e m, t r a c}(t)$ is the power to the driveline and $\omega_{\text {em,aux,min }}(t)$ is the minimum speed of the auxiliary motor as required from the driveline transmission to obtain sufficient flow to its boost circuit. $t_{0}$ and $t_{f}$ are the starting time and final time of the drive cycle, respectively. The cost to minimise is the total energy consumed by the battery during the cycle:

$$
E_{B}=\int_{t_{0}}^{t_{f}}-P_{B, c h}(t) d t
$$

where $P_{B, c h}(t)$ is the battery charging power $\left(P_{B, c h}>0\right.$ for charging, $P_{B, c h} \leq 0$ for discharging).

\section{Model}

The DP algorithm uses a backward-facing simulation model to evaluate the cost-to-go at each time step. The input to this model is thus the drive cycle, $\bar{\Lambda}(t)$, and the grid of states and control signals. In the following equations, this means that $\omega_{\text {em,aux }}$ is regarded as given.

Some important modelling assumptions:

1. Constant tank pressure, $p_{T} \approx 0$.

2. Each control valve $\left(V_{1,2,3}\right)$ and on/off valve in the dynamic valve block yield a constant pressure drop $\Delta p_{v}$.

3. Lossless cylinders.

4. When active, the bypass valves are controlled so that the full piston rod chamber flow is supplied by the piston chamber flow.

5. The pumplets' displacements are continuously variable.

6. Lossless static valve block. 


\subsection{Cylinders}

The steering motion of the machine is actuated with two identical asymmetric cylinders connected in parallel. This arrangement is modelled as an equivalent symmetric cylinder. Similarly, the boom function's two cylinders are modelled as an equivalent asymmetric cylinder. Assumptions $1-3$ yield the load pressure $p_{L, i}$ for load $i$ :

$$
p_{L, i}= \begin{cases}\frac{F_{L, i}+\Delta p_{v} A_{A, i}}{A_{B, i}}+\Delta p_{v} & P_{L, i}>0, v_{L, i}>0 \\ \frac{\Delta p_{v} A_{A, i}}{A_{B, i}}+\Delta F_{L, i} & P_{L, i}>0, v_{L, i} \leq 0 \\ \Delta p_{v} & P_{L, i} \leq 0\end{cases}
$$

where $P_{L, i}$ is the power consumed by load $i$ :

$$
P_{L, i}=F_{L, i} v_{L, i}
$$

The load flow $q_{L, i}$ for load $i$ is:

$$
q_{L, i}= \begin{cases}v_{L, i} A_{A, i} & v_{L, i}>0 \\ -v_{L, i} A_{B, i} & v_{L, i} \leq 0\end{cases}
$$

For the boom and bucket functions, assumption 4 yields:

$$
q_{L, i}=0 \text { for } P_{L, i} \leq 0, v_{L, i} \leq 0, i \in[2,3]
$$

\subsection{Dynamic valve block}

The configuration of the dynamic valve block is a function of the control signal $u_{s}$, which is implicitly defined by $\omega_{e m, a u x}$ after the introduction of the following rules:

1. Flow sharing is only used when a group is saturated,

2. When flow is shared, the load flow is divided equally between the saturated and shared pumplet groups,

3. The flow of each pumplet group is divided equally between the pumplets connected to this group,

To decide if sharing should occur, the maximum flow available at each group can first be calculated as:

$$
\bar{q}_{G, \max }=\left[\begin{array}{c}
q_{G, \max , 1} \\
\vdots \\
q_{G, \max , z_{G}}
\end{array}\right]=\bar{M}_{P G, q} \bar{q}_{p, \max }=\bar{M}_{P G, q}\left[\begin{array}{c}
q_{p, \max , 1} \\
\vdots \\
q_{p, \max , z_{p}}
\end{array}\right]
$$

where $z_{G}$ is the total number of groups and $z_{p}$ is the total number of pumplets. $\bar{M}_{P G, q}=\bar{M}_{G P, p}^{T}$ is a $\left[z_{G} \times z_{p}\right]$ matrix with zeros and ones that correspond to the connections in the static valve block (see further details in section 4.3). For the considered concept:

$$
q_{p, \max , k}= \begin{cases}D_{p} \omega_{P, 1} & 1 \leq k \leq 4 \\ D_{p} \omega_{P, 2} & 5 \leq k \leq 8\end{cases}
$$

where $\omega_{P, l}$ is the shaft speed of pump $l$ :

$$
\omega_{P, l}=i_{g, l} \omega_{e m, a u x}
$$

where $i_{g, l}$ is the gear ratio of the gear connecting pump $l$ to the auxiliary electric motor. Define $j_{\text {sat }}$ as the group index at which group flow is saturated:

$$
q_{L, j_{s a t}}>q_{G, \max , j_{s a t}}, j_{s a t} \in\left[1,2, \ldots, z_{L}\right]
$$

where $z_{L}$ is the total number of loads. According to rule 2 the flow, $q_{G, j}$, at group $j$ is then determined as: 


$$
q_{G, j}=\left\{\begin{array}{ll}
q_{L, i} & i \neq j_{s a t} \\
\frac{\sum_{i=1}^{z p} \bar{M}_{G P, p}(i, j)}{\sum_{i=1}^{z p} \bar{M}_{G P, p}(i, j)+\sum_{i=1}^{2 p} \bar{M}_{G P, p}\left(i, z_{G}\right)} \cdot q_{L, i} & i=j_{s a t}
\end{array}, j \in\left[1,2, \ldots, z_{L}\right]\right.
$$

For the considered configuration:

$$
\frac{\sum_{i=1}^{z_{p}} \bar{M}_{G P, p}(i, j)}{\sum_{i=1}^{z_{p}} \bar{M}_{G P, p}(i, j)+\sum_{i=1}^{z_{p}} \bar{M}_{G P, p}\left(i, z_{G}\right)}=\frac{1}{2}, j \in\left[1,2, \ldots, z_{L}\right]
$$

The flow at the shared group is:

$$
q_{G, 4}= \begin{cases}0 & i \neq j_{s a t} \\ q_{L, i}-q_{G, i} & i=j_{s a t}\end{cases}
$$

and finally the group pressures are:

$$
p_{G, j}=\left\{\begin{array}{lll}
p_{L, j} & j \in[1,2,3] \\
\{ & i \neq j_{\text {sat }} & j=4 \\
p_{L, i}+\Delta p_{v} & i=j_{\text {sat }}
\end{array}\right.
$$

\subsection{Static Valve Block}

To determine the pumplet flows and pressures, the group flows and pressures are first collected in the vectors $\bar{q}_{G}$ and $\bar{p}_{G}$, respectively:

$$
\bar{q}_{G}=\left[\begin{array}{c}
q_{G, 1} \\
\vdots \\
q_{G, z_{G}}
\end{array}\right], \bar{p}_{G}=\left[\begin{array}{c}
p_{G, 1} \\
\vdots \\
p_{G, z_{G}}
\end{array}\right]
$$

Similarly, the pumplet flows and pressures are collected as:

$$
\bar{q}_{p}=\left[\begin{array}{c}
q_{p, 1} \\
\vdots \\
q_{p, z_{p}}
\end{array}\right], \bar{p}_{p}=\left[\begin{array}{c}
p_{p, 1} \\
\vdots \\
p_{p, z_{p}}
\end{array}\right]
$$

Ignoring pressure losses in the static valve bock, the pumplet pressures can then be determined by:

$$
\bar{p}_{p}=\bar{M}_{G P, p} \cdot \bar{p}_{G}
$$

where $\bar{M}_{G P, p}$ is a $\left[z_{p} \times z_{G}\right]$ matrix with ones and zeros that corresponds to the connections in the static valve block. For the configuration considered in this paper (figure 3):

$$
\bar{M}_{G P, p}=\left[\begin{array}{cccc}
1 & 0 & 0 & 0 \\
1 & 0 & 0 & 0 \\
0 & 1 & 0 & 0 \\
0 & 1 & 0 & 0 \\
0 & 0 & 1 & 0 \\
0 & 0 & 1 & 0 \\
0 & 0 & 0 & 1 \\
0 & 0 & 0 & 1
\end{array}\right]
$$

Ignoring leakage and obeying rule 3, the pumplet flows are determined as:

$$
\bar{q}_{p}=\bar{M}_{G P, q} \cdot \bar{q}_{G}
$$

where $\bar{M}_{G P, q}$ is a $\left[z_{G} \times z_{p}\right]$ matrix with each element: 


$$
\bar{M}_{G P, q}(k, j)=\frac{\bar{M}_{G P, p}(k, j)}{\sum_{i=1}^{z_{p}} \bar{M}_{G P, p}(i, j)}
$$

For the configuration considered in this paper:

$$
\bar{M}_{G P, q}=\frac{1}{2} \cdot \bar{M}_{G P, p}
$$

\subsection{Pumps}

The relative displacement, $\varepsilon_{p, k}$, of pumplet $k$ connected to pump $l$ can then be determined according to:

$$
\varepsilon_{p, k}=\frac{q_{p, k}}{D_{p} \omega_{P, l}}
$$

The total efficiency, $\eta_{p, k}$, of pumplet $k$ connected to pump $l$ is then calculated with linear interpolation in an efficiency map obtained from the pump manufacturer:

$$
\eta_{p, k}=f\left(\omega_{P, l}, p_{p, k}, \varepsilon_{p, k}\right)
$$

The efficiencies are then used to calculate the input power to each pumplet:

$$
P_{p, k}=\frac{q_{p, k} p_{p, k}}{\eta_{p, k}}
$$

The power input to each pumplet can then be used to calculate the torques of pump 1 and 2:

$$
T_{P, 1}=\frac{\sum_{k=1}^{4} P_{p, k}}{\omega_{P, 1}}, T_{P, 2}=\frac{\sum_{k=5}^{z_{p}} P_{p, k}}{\omega_{P, 2}}
$$

\subsection{Mechanical Losses}

The boost circuit for the driveline transmission is modelled as a constant torque, assuming ideal pressure relief valve characteristics:

$$
T_{b, t r a c}=\frac{p_{b} D_{b}}{\eta_{p, b}}
$$

where $p_{b}$ is the boost pressure, $D_{b}$ the boost pump volumetric displacement and $\eta_{p, b}$ the boost pump hydromechanical efficiency (assumed constant, $\eta_{p, b} \approx 0.8$ ). Other peripheral mechanical losses are modelled as a constant power loss, estimated from the drive cycles:

$$
T_{p e r, M}=\frac{P_{p e r, M}}{\omega_{P, 2}}
$$

\subsection{Auxiliary Electric Motor}

The total torque on the auxiliary electric motor is then:

$$
T_{e m, a u x}=\frac{T_{P, 1}+T_{b, t r a c}}{\eta_{g}} i_{g, 1}+\frac{T_{P, 2}+T_{p e r, M}}{\eta_{g}} i_{g, 2}
$$

where $\eta_{g}$ are the gear efficiencies (assumed constant, $\eta_{g} \approx 0.98$ ). The efficiency, $\eta_{e m, a u x}$, of the auxiliary electric motor is calculated with linear interpolation in an efficiency map obtained from the electric motor manufacturer:

$$
\eta_{e m, a u x}=f\left(T_{e m, a u x}, \omega_{e m, a u x}\right)
$$

which yields the input power to the auxiliary electric motor:

$$
P_{e m, a u x, i n}=\frac{T_{e m, a u x} \omega_{e m, a u x}}{\eta_{e m, a u x}}
$$




\subsection{Electric circuit}

The battery output power is calculated as:

$$
P_{B}=-P_{e m, t r a c}-\frac{P_{e m, a u x, i n}}{\eta_{i n v}}-P_{p e r, E}
$$

where $P_{p e r, E}$ is electric peripheral losses, which are assumed constant and were estimated from the drive cycle. $\eta_{i n v}$ is the inverter efficiency (assumed constant). $P_{e m, t r a c}$ is the power to the driveline electric motor:

$$
P_{\text {em }, \text { trac }}= \begin{cases}\frac{P_{\text {trac }}}{\eta_{\text {inv }}} & P_{\text {trac }} \geq 0 \\ P_{\text {trac }} \eta_{\text {inv }} & P_{\text {trac }}<0\end{cases}
$$

where $P_{t r a c}$ is the power to the driveline recorded during the drive cycle. The battery current, $I_{B}$, is then obtained as:

$$
I_{B}=\frac{-U_{e m k}}{2 R_{B}}+\sqrt{\left(\frac{U_{e m k}}{2 R_{B}}\right)^{2}+\frac{P_{B}}{R_{B}}}
$$

where $U_{e m k}$ and $R_{B}$ are the electromotive force voltage and the internal resistance of the battery, respectively, which are both assumed to be constant and which values were obtained from the battery manufacturer. This finally yields the battery charge power:

$$
P_{B, c h}=P_{B}-R_{B} I_{B}^{2}
$$

\section{Drive Cycle}

The drive cycle used in the optimisation is a short loading cycle (two repetitions, with total time $t_{f}=140$ seconds, $t_{0}=0$ ) that was recorded with a ST14 Battery operated by a professional operator. In the recorded cycle, the machine fills the bucket in a gravel pile, reverses, turns, drives to another pile and empties the bucket. After the recording, the results were post-processed to obtain some of the states used in the optimisation (see equation (2)). The piston forces were calculated from the logged cylinder pressures and the cylinder dimensions, assuming lossless cylinders. The boom piston position was first calculated from the logged boom angle and geometrical data and then differentiated and filtered with a moving average filter to obtain the piston velocity. Similarly, the steer piston velocity was obtained from the steer angle while the bucket velocity was calculated from the logged piston position. The driveline power was calculated from the logged torque and speed of the driveline electric motor.

\section{Results and Discussion}

The optimisation problem in section 3 was solved with DP using the implementation in [10] with the settings in table 1 . The time discretisation was made with a time step of 0.2 seconds, which is regarded as the minimum actuation time of the on/off valves used in the dynamic valve block.

Table 1: Optimisation settings.

\begin{tabular}{lccc}
\hline Parameter & & Value & Unit \\
\hline Time step & & 0.2 & $\mathrm{~seconds}$ \\
Max motor speed & $\omega_{\text {em,aux,max }}$ & 3000 & $\mathrm{rpm}$ \\
Motor speed grid size & & 200 & $\mathrm{points}$ \\
Minimum motor shaft acceleration & $u_{\text {em,min }}$ & -100 & $\mathrm{rad} / \mathrm{s}^{2}$ \\
Maximum motor shaft acceleration & $u_{\text {em,max }}$ & 100 & $\mathrm{rad} / \mathrm{s}^{2}$ \\
Motor shaft acceleration grid size & & 49 & $\mathrm{points}$ \\
\hline
\end{tabular}

The resulting state trajectory (auxiliary motor shaft speed), the shared group load dedication $\left(u_{s}(t)\right)$ and the pumplet group pressures for the optimised cycle are shown in figure 4 while the group and load flows are shown in figure 5 . The optimisation maximises the shared group use to minimise the auxiliary motor speed. Parts of the cycle require higher shaft speed to match the required flow. These parts can be observed in figures 4-5 as the bucket filling phases (20-40 seconds and 80-100 seconds) and the boom raising phases (50-70 seconds and 110-120 seconds). 


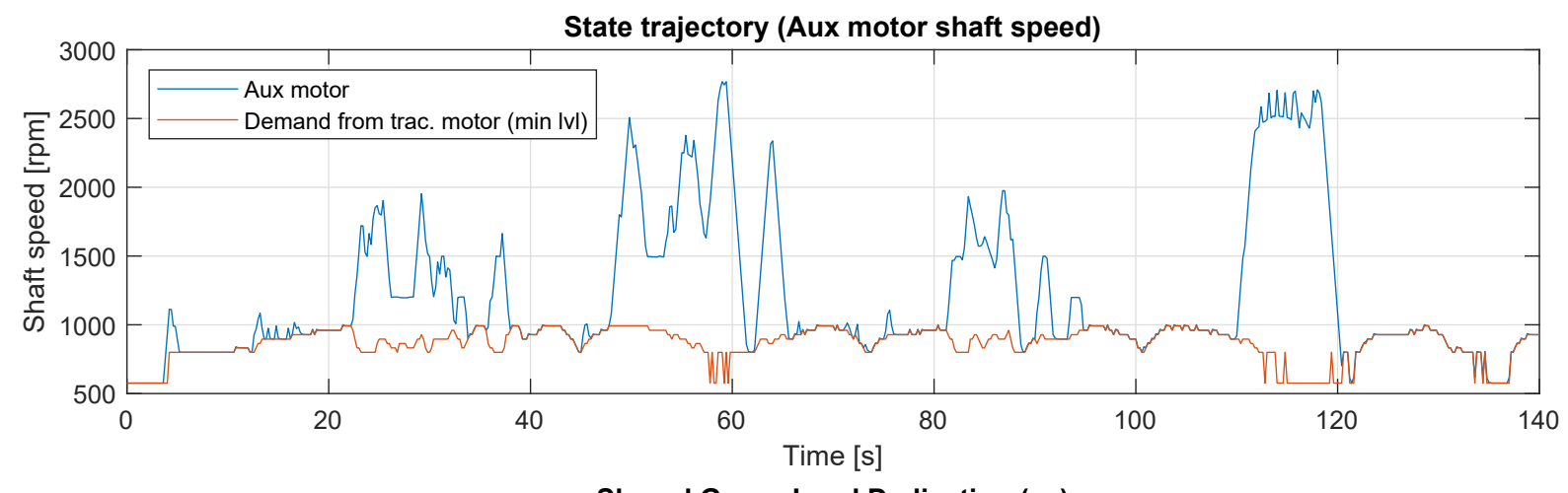

Shared Group Load Dedication $\left(u_{s}\right)$

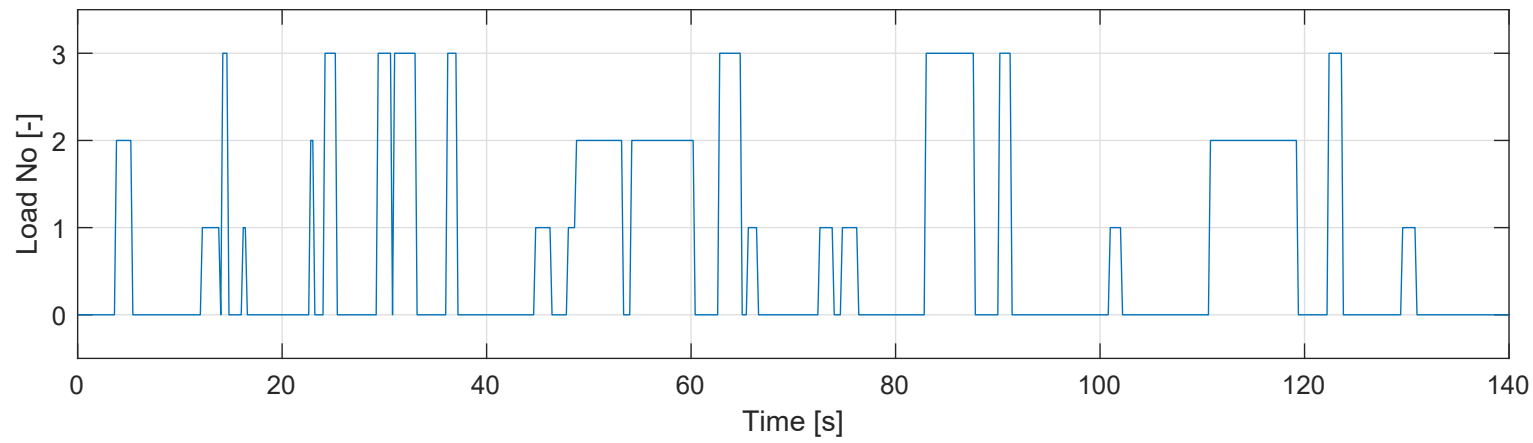

Group pressures

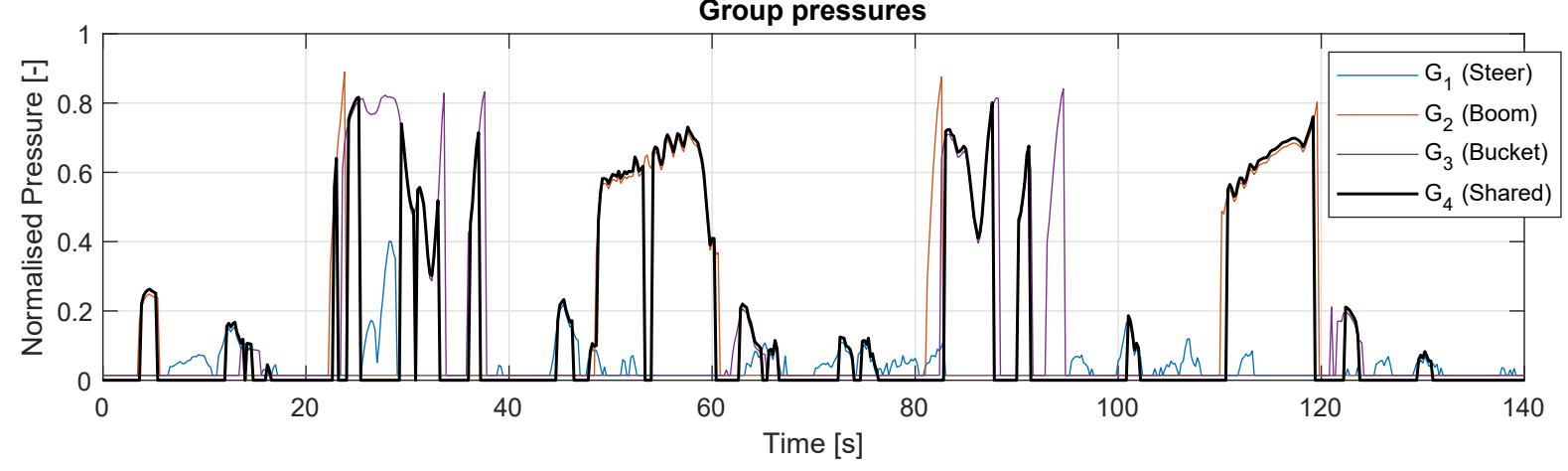

Figure 4: Auxiliary motor speed, flow sharing control signal and normalised group pressures for the optimised cycle.

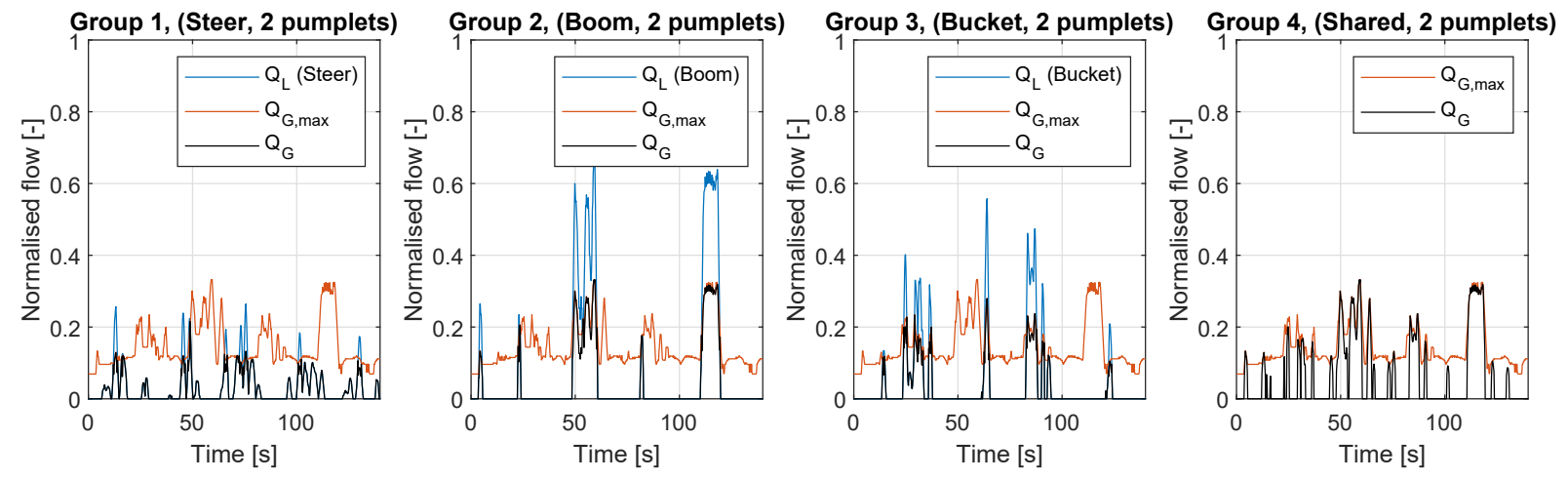

Figure 5: Normalised group and load flows for the optimised cycle. 
Figure 6 shows bubble plots for the pumps and the auxiliary electric motor. In these figures as well, it is clear that low speed is preferred, since around $50 \%$ of the cycle is spent near $900 \mathrm{rpm}$. It may be noted though, that maximum efficiency of the pumps and the electric motors is not prioritised, as the efficiency-optimal speeds for both these components are significantly higher (around 2000-3500 rpm). It was found that the reason for this was the power loss due to the transmission boost circuit. This loss increases proportionally to the electric motor shaft speed and is significantly larger (approximately a factor 2) than the losses in the pumps and the electric motor. These results highlight the benefits of considering the complete system rather than the individual components when evaluating energy efficiency, and of having high and relatively flat (operation point-independent) efficiencies in the pumps and electric motors. For the ST14 Battery machine, the results also suggest that an alternative solution for the boost circuit could be interesting.

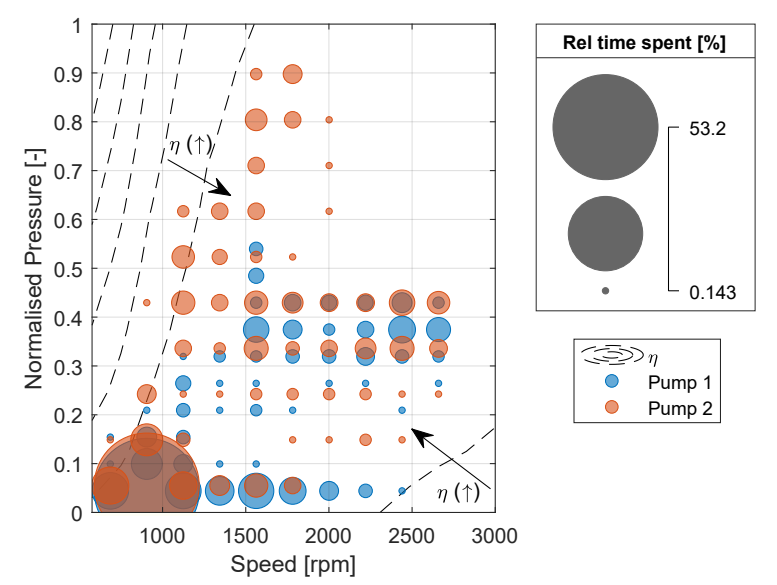

(a) Pumps. The mean values of the pumplet pressures have been used. The efficiency map is shown for $50 \%$ of full pump displacement.

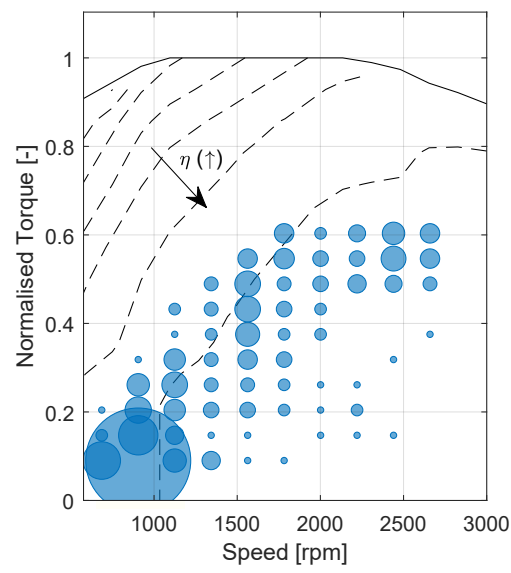

(b) Electric motor.
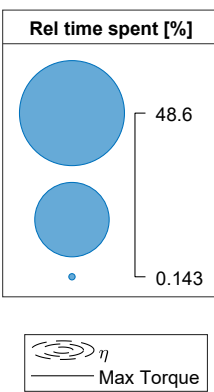

Figure 6: Bubble plots for the pumps and the auxiliary electric motor for the optimised cycle.

To investigate the gains of optimally controlling the motor speed, an optimisation with constant speed, chosen as the highest value in figure $4(2800 \mathrm{rpm})$, was carried out. The resulting cycle energy is shown in figure 7 , where the energy for the baseline case (load-sensing with axial-piston pumps) and the optimised case are also shown for comparison. Compared with the baseline case, the total cycle energy was decreased with $5 \%$ using constant speed, while optimal speed control added an additional drop of $4 \%$. The losses decreased with $16 \%$ with constant speed with an additional drop of $14 \%$ with optimally controlled speed. The primary cause for the improvements were found to be reduced pump losses and reduced valve throttling losses. One important source of error are the peripheral electrical losses $\left(P_{p e r, E}\right)$, which were difficult to estimate accurately and stands for approximately $7-8$ $\%$ of the cycle's total energy consumption. This indicates a need to investigate these losses in more detail.

Compared to [6,7], the obtained improvements may be considered surprisingly small. In [6,7], the application is, however, an excavator with a diesel engine, where parts of the savings were achieved by selecting a more fueloptimal point of the engine. In addition, all power is actuated with the hydraulic system in an excavator while the driveline represented $53 \%$ of the energy consumption for the scooptram in the considered cycle (see figure 7a). Moreover, in contrast to an excavator, simultaneous use of multiple hydraulic functions is rare in a scooptram, which results in low pressure-compensating losses in the baseline case. The gains from introducing pump control in the considered application are therefore relatively low compared to the excavator considered in [7].

The results indicate that approximately $4 \%$ extra energy savings are gained by optimally controlling the electric motor speed, where the gains are almost exclusively due to the lowered losses in the transmission boost circuit. This improvement is in the same order of magnitude as was found for the truck loader crane considered in [8], and may be seen as moderate. One important reason for this improvement being moderate is that the pumps and the electric motor both have relatively high efficiency for a large operational domain, which therefore makes the speed parameter of less importance (efficiency-wise) for these components. The fact that scooptrams often operate non-stop in short loading cycles still, however, makes $4 \%$ a desirable improvement. On that same topic, all the presented results are based on one cycle, and other cycles may lead to a different number.

Furthermore, it may be noted that the boom and bucket functions have high amounts of energy that potentially be recuperated, which could further lower the energy losses. 


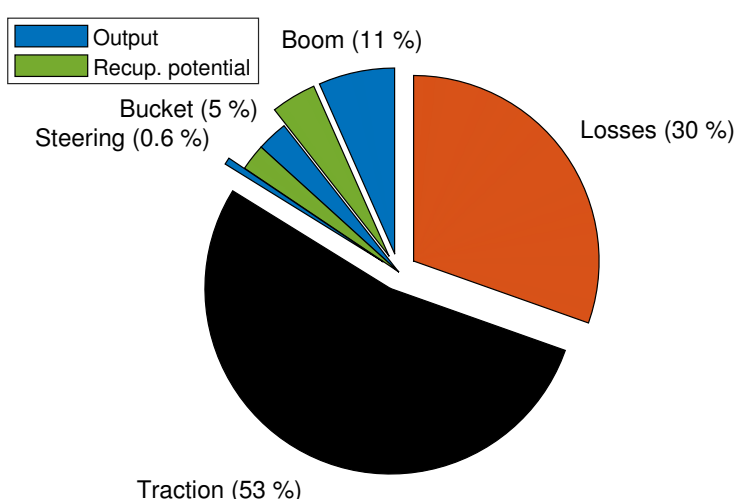

(a) Cycle energy distribution (Baseline). The function energies were calculated as the integrated mechanical power $\left(F_{L, i} \cdot v_{L, i}\right)$ for each function. The green fields indicate negative power that potentially could be recuperated. The losses were calculated as the difference between the logged battery input power and the total output power (functions plus traction).

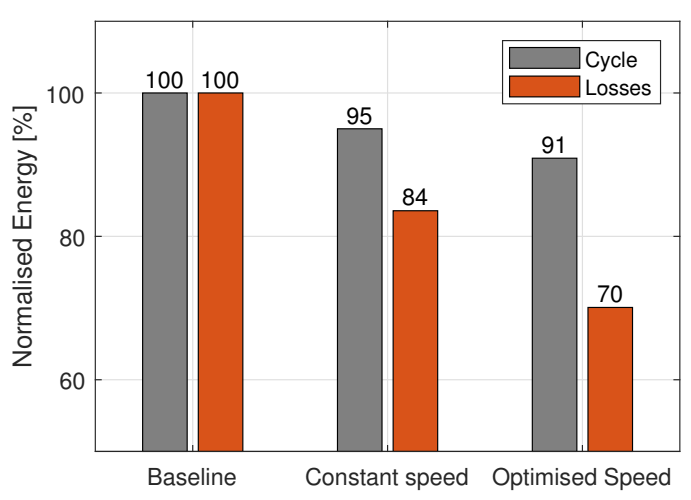

(b) Cycle energy and energy losses for different cases.

Figure 7: Cycle energy. The baseline cycle energy was calculated from the recorded battery current and voltage.

\section{Conclusions and Outlook}

The Digital Displacement Pump facilitates the implementation of pump control in a mobile working machine. By using control optimisation, the maximum potential of the considered pump control concept with dynamic flow sharing could be evaluated. The optimal control results show that a strategy with minimised electric motor speed is preferred, primarily due to drag losses from the boost circuit of the transmission clutch actuation system. This strategy is facilitated by the high and relatively operation point-independent efficiencies of the pumps and electric motor. The results indicate a $5 \%$ decrease in total system energy use for the considered short loading cycle, with an additional $4 \%$ drop if the electric motor speed is optimally controlled. The improvements primarily come from reduced pump and valve throttling losses.

The low amount of simultaneous function use in the considered application suggest that greater loss reductions is to be expected for a more hydraulic-intense application, such as an excavator. Moreover, the obtained results depend on the considered concept, load cycle and the component sizes. It should also be emphasised that the obtained optimal strategy is not implementable in a causal controller, which indicate lower savings in a final application. Other applications, load cycles, concepts, combined control and component sizing optimisation and development of causal control strategies are, consequently, subjects for future work.

\section{Acknowledgements}

This research was funded by the Swedish Energy Agency (Energimyndigheten, Grant Number 50181-2), Volvo Construction Equipment $\mathrm{AB}$ and Epiroc $\mathrm{AB}$. The authors would particularly like to thank Simon Magnusson and Markus Bagge at Eiproc for their input concerning the ST14 Battery machine, and Kim Heybroek at Volvo Construction Equipment for valuable input on working machines.

\section{Nomenclature}

\begin{tabular}{lll}
\hline \multicolumn{2}{l}{ Designation Denotation } & Unit \\
\hline$A_{A / B}$ & A/B-side piston area & $\mathrm{m}^{2}$ \\
$D$ & Volumetric displacement & $\mathrm{m}^{3} / \mathrm{rad}$ \\
$\Delta p_{v}$ & Valve pressure drop & $\mathrm{Pa}$ \\
$E$ & Energy & $\mathrm{J}$ \\
$\varepsilon$ & Relative displacement & - \\
$\eta$ & Efficiency & - \\
$F$ & Force & $\mathrm{N}$ \\
$I$ & Current & $\mathrm{A}$ \\
$i_{g}$ & Gear ratio & - \\
$\Lambda$ & Drive cycle & (vector) \\
\hline
\end{tabular}




\begin{tabular}{|c|c|c|c|c|c|}
\hline $\bar{M}_{G P, p / q}$ & \multicolumn{4}{|c|}{ Static valve block pressure/flow matrix (Groups->pumplets) } & (matrix) \\
\hline $\bar{M}_{G P, p / q}$ & \multicolumn{4}{|c|}{ Static valve block pressure/flow matrix (Groups->pumplets) } & (matrix) \\
\hline$v$ & \multicolumn{4}{|c|}{ Velocity } & $\mathrm{m} / \mathrm{s}$ \\
\hline$E$ & \multicolumn{4}{|l|}{ Energy } & $\mathrm{J}$ \\
\hline$\omega$ & \multicolumn{4}{|l|}{ Rotational speed } & $\mathrm{rad} / \mathrm{s}$ \\
\hline$P$ & \multicolumn{4}{|l|}{ Power } & W \\
\hline$p$ & \multicolumn{4}{|l|}{ Pressure } & $\mathrm{Pa}$ \\
\hline$q$ & \multicolumn{4}{|l|}{ Flow } & $\mathrm{m}^{3} / \mathrm{s}$ \\
\hline$R$ & \multicolumn{4}{|l|}{ Resistance } & Ohm \\
\hline$T$ & \multicolumn{4}{|l|}{ Torque } & $\mathrm{Nm}$ \\
\hline$t_{0}$ & \multicolumn{4}{|l|}{ Cycle start time } & seconds \\
\hline$t_{f}$ & \multicolumn{4}{|l|}{ Cycle end time } & seconds \\
\hline$U$ & \multicolumn{4}{|l|}{ Voltage } & $\mathrm{V}$ \\
\hline$u_{e m / s}$ & \multicolumn{4}{|c|}{ Electric motor/flow sharing control signal } & $\mathrm{rad} / \mathrm{s}^{2}(-)$ \\
\hline$z_{G}$ & \multicolumn{4}{|c|}{ Total number of groups $\left(z_{G}=4\right.$ here $)$} & - \\
\hline$z_{L}$ & \multicolumn{4}{|c|}{ Total number of loads $\left(z_{L}=3\right.$ here $)$} & - \\
\hline$z_{p}$ & \multicolumn{4}{|c|}{ Total number of pumplets ( $z_{p}=8$ here) } & - \\
\hline \multicolumn{6}{|c|}{ Subscripts } \\
\hline aux & Auxiliary & $i$ & Load number & $P$ & Pump \\
\hline$B$ & Battery & inv & Inverter & $p$ & Pumplet \\
\hline$b$ & Boost & $j$ & Group number & per & Peripheral \\
\hline$E$ & Electric & $k$ & Pumplet number & sat & Saturated \\
\hline em & Electric motor & $L$ & Load & $T$ & Tank \\
\hline emk & Electromotive force & $l$ & Pump number & trac & Traction \\
\hline$G$ & Group & $M$ & Mechanical & & \\
\hline
\end{tabular}

\section{References}

[1] Søren Ketelsen, Damiano Padovani, Torben O. Andersen, Morten Kjeld Ebbesen, and Lasse Schmidt. Classification and review of pump-controlled differential cylinder drives. Energies, 12(7), 2019.

[2] Kim Heybroek. On Energy Efficient Mobile Hydraulic Systems: with Focus on Linear Actuation. PhD thesis, Linköping University, 2017.

[3] Danfoss Power Solutions. Digital Displacement Pump, User Guide https://assets.danfoss.com/ documents/149654/BC306384089197en-000102.pdf. Last visited on: 2021-02-17.

[4] Epiroc. Scooptram ST14 Battery http://www.podshop.se/Epiroc/epiroc/Products/ DownloadLowres $/$ ?productRef $=83685$. Last visited on: 2021-02-17.

[5] Niall Caldwell. Review of Early Work on Digital Displacement ${ }^{\circledR}$ Hydrostatic Transmission Systems. volume BATH/ASME 2018 Symposium on Fluid Power and Motion Control of Fluid Power Systems Technology, 09 2018. V001T01A061.

[6] Matthew Green, Jill Macpherson, Niall Caldwell, and W. H. S. Rampen. DEXTER: The Application of a Digital Displacement ${ }^{\circledR}$ Pump to a 16 Tonne Excavator. volume BATH/ASME 2018 Symposium on Fluid Power and Motion Control of Fluid Power Systems Technology, 092018.

[7] Matteo Pellegri, Matthew Green, Jill Macpherson, Callan McKay, and Niall Caldwell. Applying a MultiService Digital Displacement ${ }^{\circledR}$ Pump to an Excavator to Reduce Valve Losses. In 12th International Fluid Power Conference (IFK'20), Dresden, Germany, 2020.

[8] Samuel Kärnell, Amy Rankka, Alessandro Dell'Amico, and Liselott Ericson. Digital Pumps in SpeedControlled Systems - An Energy Study for a Loader Crane Application. In 12th International Fluid Power Conference (IFK'20), Dresden, Germany, 2020.

[9] Michael Sprengel and Monika Ivantysynova. Neural network based power management of hydraulic hybrid vehicles. International Journal of Fluid Power, 18(2):79-91, 2017.

[10] Olle Sundstrom and Lino Guzzella. A generic dynamic programming matlab function. In 2009 IEEE Control Applications, (CCA) Intelligent Control, (ISIC), pages 1625-1630, 2009. 